\title{
Acidic Polyamino Acids Inhibit Human Eosinophil Granule Major Basic Protein Toxicity

\author{
Evidence of a Functional Role for ProMBP
}

\author{
Robert L. Barker, Robert H. Gundel,“ Gerald J. Gleich, James L. Checkel, \\ David A. Loegering, Larry R. Pease, and Kimm J. Hamann \\ Department of Immunology and the Allergic Diseases Research Laboratory, Mayo Clinic and Mayo Foundation, and the Mayo Medical \\ School, Rochester, Minnesota 55905; and *Department of Pharmacology, Boehringer Ingelheim Pharmaceuticals, Inc., Ridgefield, \\ Connecticut 06877
}

\begin{abstract}
Eosinophil granule major basic protein (MBP), a potent toxin for helminths and mammalian cells in vitro, is a single polypeptide chain rich in arginine. MBP has been localized on damaged helminths and tissues in hypersensitivity diseases including bronchial asthma. The MBP cDNA indicates that MBP is translated as a slightly acidic preproprotein with an acidic propart. To test the hypothesis that the acidic pro-part of proMBP inhibits the toxicity of mature MBP, acidic polyamino acids (aa) were used as antagonists of MBP toxicity to $\mathrm{K562}$ cells and guinea pig tracheal epithelium and used as antagonists of MBP airway hyperresponsiveness in primates. The acidic poly aa inhibited MBP toxicity and MBP airway hyperresponsiveness. The acidic poly aa inhibited MBP toxicity in a charge-dependent manner similar to that proposed for proMBP, suggesting that the acidic pro-part of proMBP functions to mask mature MBP toxicity. This inhibition was not limited to MBP, but also applied to polyarginine and eosinophil cationic protein. These acidic poly aa may be useful to inhibit the actions of a number of cationic toxins released by the eosinophil in numerous hypersensitivity diseases. (J. Clin. Invest. 1991. 88:798-805.) Key words: bronchial asthma • airway hyperresponsiveness • polyarginine $\bullet$ eosinophil cationic protein $\bullet$ anionic polymers
\end{abstract}

\section{Introduction}

The eosinophilic leukocyte contains a number of cationic toxins. Major basic protein (MBP), ${ }^{1}$ the predominant protein in eosinophils, comprises the crystalloid core of the eosinophil granule (1) and in humans is a 117-amino acid (aa) residue

Address reprint requests to Kimm J. Hamann, Ph.D., Section of Pulmonary and Critical Care Medicine, University of Chicago, Box 98, 5841 South Maryland Avenue, Chicago, IL 60637.

Received for publication 31 January 1991 and in revised form 13 May 1991.

1. Abbreviations used in this paper: aa, amino acid; DCS, defined calf serum; e, electronic charge; ECP, eosinophil cationic protein; MBP, major basic protein; metacholine $P_{100}$, the dose of inhaled metacholine required to cause a $100 \%$ increase in Rrs; Rrs, respiratory system resistance.

J. Clin. Invest.

(c) The American Society for Clinical Investigation, Inc. 0021-9738/91/09/0798/08 \$2.00

Volume 88, September 1991, 798-805 single polypeptide rich in arginine (2). MBP likely damages cell membranes $(3,4)$ and is a potent toxin for helminths and mammalian cells in vitro (1). It has been localized on damaged helminths and tissues in hypersensitivity diseases including bronchial asthma. MBP causes extensive damage to guinea pig and human respiratory epithelium; this damage mimics the pathology of asthma, suggesting that eosinophils are a prime effector cell for tissue damage in bronchial asthma (1). MBP instillation into the trachea of primates results in a significant increase in airway responsiveness, indicating a direct role for MBP in the pathogenesis of airway hyperresponsiveness (5).

The MBP cDNA from the promyelocytic cell line HL-60, which produces MBP, indicates that MBP is translated as a slightly acidic preproprotein with an acidic pro-part (6). Human MBP (2) contains a high portion of aa with hydrophobic side chains, 17 arginines, only one carboxylic acid-containing residue, and has a calculated electronic charge (e) (calculated by Titrate program, DNAstar, Inc., Madison, WI) of 16.3 (7). The 90-aa residue acidic pro-part of proMBP contains 20 glutamic acids, 6 aspartic acids, 7 strongly basic aa, and has a calculated e of -19.0 . The combination of the acidic and basic peptides in proMBP has an almost neutral $\mathrm{e}$ of -2.58 . Previously, we suggested that the acidic pro-part of proMBP masks the toxic effects of mature MBP and protects the eosinophil from damage while the protein is processed through the endoplasmic reticulum to its sequestered site in the eosinophil granule as toxic MBP (6).

To test the hypothesis that the acidic pro-part of proMBP inhibits mature MBP toxicity, acidic poly aa were used as substitutes for the acidic pro-part of proMBP which has not yet been isolated in its native state or obtained in a recombinant form. Here, we report that acidic poly aa inhibited MBP toxicity to K562 cells and guinea pig tracheal epithelium and inhibited MBP airway hyperresponsiveness in primates. Acidic poly aa inhibited MBP toxicity in a charge-dependent manner similar to that proposed for proMBP, providing evidence that the acidic pro-part of proMBP functions to neutralize toxic MBP and suggesting a possible therapy for bronchial asthma.

\section{Methods}

\section{Reagents and solutions}

Amino acids, amino acid polymers (Table I), RPMI 1640 medium containing L-glutamine and methacholine were purchased from Sigma Chemical Co., St. Louis, MO. Defined calf serum (DCS) was obtained from Hyclone Laboratories, Inc., Logan, UT. Ketamine (Ketaset) and xylazine (Rompun) were purchased from Bristol Laboratories, Evansville, IN, and Miles Inc., West Haven, CT, respectively. 
Table I. Selected Properties of AA and Proteins Used in This Study

\begin{tabular}{|c|c|c|c|c|}
\hline & Mol wt & $\begin{array}{c}\text { Degree of } \\
\text { polymerization* }\end{array}$ & $\begin{array}{l}\text { Charge (e) } \\
\text { at pH } 7.0^{\ddagger}\end{array}$ & $\begin{array}{l}\text { Concentration for } \\
\text { balanced charge }\end{array}$ \\
\hline L-aspartic acid (A-0651)" & 155 & 1 & -1.1 & (a) $7.5 \times 10^{-5} \mathrm{M}$ \\
\hline L-glutamic acid salt (G-1626) & 169 & 1 & -1.1 & (a) $7.5 \times 10^{-5} \mathrm{M}$ \\
\hline Poly-( $\alpha, \beta)$-DL-aspartic acid (P-3418) & 6,800 & 50 & -50.1 & (a) $1.6 \times 10^{-6} \mathrm{M}$ \\
\hline Poly (aspartic acid, glutamic acid) 1:1 (P-1408) & 9,000 & 60 & -60.0 & (a) $1.4 \times 10^{-6} \mathrm{M}$ \\
\hline Poly-L-aspartic acid (P-5387) & 11,500 & 84 & -84.0 & (a) $9.7 \times 10^{-7} \mathrm{M}$ \\
\hline Poly-L-glutamic acid (P-4636) & 13,600 & 90 & -89.9 & $\begin{array}{l}\text { (a) } 9.1 \times 10^{-7} \mathrm{M} \\
\text { (b) } 3.3 \times 10^{-6} \mathrm{M} \\
\text { (c) } 8.1 \times 10^{-7} \mathrm{M}\end{array}$ \\
\hline Polyglutamic acid (P-4761) & 36,240 & 240 & -239.0 & (a) $3.4 \times 10^{-7} \mathrm{M}$ \\
\hline Poly-D-glutamic acid (P-4033) & 41,000 & 272 & -271.0 & (a) $3.0 \times 10^{-7} \mathrm{M}$ \\
\hline Poly-L-aspartic acid (P-6762) & 42,500 & 310 & -310.0 & (a) $2.6 \times 10^{-7} \mathrm{M}$ \\
\hline Poly-D-glutamic acid (P-4637) & 66,000 & 437 & -436.0 & (a) $1.9 \times 10^{-7} \mathrm{M}$ \\
\hline Poly-L-glutamic acid (P-4886) & 77,800 & 515 & -514.0 & $\begin{array}{l}\text { (a) } 1.6 \times 10^{-7} \mathrm{M} \\
\text { (b) } 5.9 \times 10^{-7} \mathrm{M} \\
\text { (c) } 1.4 \times 10^{-7} \mathrm{M}\end{array}$ \\
\hline Poly-L-asparagine (P-8137) & 10,400 & 91 & -0.1 & \\
\hline Native MBP & 13,801 & 117 & 16.3 & \\
\hline Poly-L-arginine hydrochloride (P-4663) & 12,000 & 62 & 60.9 & \\
\hline ECP & 16,000 & 133 & 14.5 & \\
\hline
\end{tabular}

* Degree of polymerization indicates number of aa residues per molecule. ${ }^{\ddagger}$ Charge (e) calculated by Titrate program (DNAstar, Inc., Madison, WI). ${ }^{8}$ Concentration of acidic aa necessary to balance charge of cationic: (a) MBP at $5 \times 10^{-6} \mathrm{M}$; (b) poly-L-arginine hydrochloride at $5 \times 10^{-6}$ $\mathrm{M}$; or (c) ECP at $5 \times 10^{-6} \mathrm{M}$. " All acids used were purchased as sodium salts; Sigma product code appears in parentheses after the product name.

\section{Purification of MBP and eosinophil cationic protein}

Eosinophils from patients with hypereosinophilic syndrome were obtained by cytapheresis as previously described $(8,9)$. The procedures for purification of human eosinophil granules, MBP, and eosinophil cationic protein (ECP) have been described in detail elsewhere $(10,11)$. Briefly, after eosinophil lysis and removal of cell debris and unbroken cells by centrifugation, granule-containing supernatants were pooled and centrifuged. Granule pellets were solubilized in $0.01 \mathrm{M} \mathrm{HCl}$ (final $\mathrm{pH} 3.0$ ) with brief sonication and centrifuged at $40,000 \mathrm{~g}$ for $5 \mathrm{~min}$. The supernatant was fractionated at $4^{\circ} \mathrm{C}$ on a Sephadex G-50 column equilibrated with $0.025 \mathrm{M}$ sodium acetate buffer ( $\mathrm{pH} 4.2$ ) containing $0.15 \mathrm{M}$ $\mathrm{NaCl}$ (column buffer). Fractions from the second protein peak containing ECP were pooled, dialyzed against PBS, lyophilized, and further purified as described below. Fractions from the third protein peak, containing only native MBP, were pooled. MBP concentrations were determined by absorbance at $277 \mathrm{~nm}$ using the extinction coefficient $\mathrm{E}_{1 \mathrm{~cm}}^{1 \%}=26.3$. ECP was further purified by affinity chromatography on a heparin-CL-6B column equilibrated with PBS at pH 7.4. Fractions containing ECP eluted from the heparin-Sepharose at high salt concentrations ( $0.15 \mathrm{M}-1.5 \mathrm{M} \mathrm{NaCl}$ gradient used) and were pooled, dialyzed against PBS, and concentrated by lyophilization. ECP concentrations were determined by absorbance at $277 \mathrm{~nm}$ using the extinction coeffcient $\mathrm{E}_{1 \mathrm{~cm}}^{1 \%}=15.6$.

\section{Cytotoxicity and inhibition of toxicity assays}

K562 cell cultures (CCL 243; American Type Culture Collection, Rockville, MD) were maintained in RPMI 1640 supplemented with L-glutamine and $10 \%$ DCS by twice-weekly passage. $2 \mathrm{~d}$ before assay, cells were resuspended at $4 \times 10^{4}$ cells/ml of fresh RPMI 1640 with $10 \%$ DCS, pH 7.4, in 50-ml flasks. By the day of assay, cell numbers had reached 1.5-2.0 $\times 10^{5} / \mathrm{ml}$. These cells were washed three times in RPMI 1640 without DCS, resuspended to $5 \times 10^{5}$ cells $/ \mathrm{ml}$, and duplicate 100- $\mu$ aliquots were dispensed to flat-bottomed 96-well microtiter plates. $50 \mu \mathrm{l}$ of various concentrations of amino acids dissolved in RPMI 1640 or RPMI 1640 alone were added followed at the appro- priate time by $50 \mu \mathrm{l}$ of MBP in column buffer, poly-L-arginine in RPMI 1640 , ECP in RPMI 1640, or appropriate controls. MBP, poly-L-arginine, and ECP were used at a final concentration of $5 \times 10^{-6} \mathrm{M}$. Cells were incubated for $4 \mathrm{~h}$ at $37^{\circ} \mathrm{C}$ in a $5 \% \mathrm{CO}_{2}$ atmosphere in a humidified chamber. Cell viability was determined by microscopic observation using trypan blue exclusion and mean percent viability \pm 1 SD of duplicates from one or more experiments was calculated. Differences in viability between nontoxic controls and test concentrations were evaluated statistically using Student's $t$ test. Nontoxic controls were used in the statistical analysis instead of the toxic control (MBP, poly-L-arginine, or ECP alone) because we wished to emphasize the point at which acidic poly aa inhibition of toxicity declined. After the initial experiments using all of the acidic poly aa, poly-L-glutamic acid 13,600 mol wt and poly-L-glutamic acid 77,800 mol wt, the lowest and highest molecular weight polyglutamic acid homopolymers, were used as typical representatives of the acidic poly aa. Optimal assay conditions for MBP toxicity to K562 cells have been previously determined (12).

\section{Guinea pig tracheal ring assay}

Tracheal rings were prepared from Hartley guinea pigs (Mayo Institute Hills Farm, Rochester, MN) as previously described (13). Briefly, immediately after $\mathrm{CO}_{2}$ euthanasia tracheas were removed and placed in RPMI 1640. Adherent connective tissue was trimmed and each trachea was cut transversely into a series of $1-\mathrm{mm}$ thick rings using a previously described slicing device (14). After overnight equilibration, each ring was carefully examined to determine that the mucosa was intact and actively beating cilia were present. Rings with damaged mucosa were not used for experimentation.

For testing, rings were transferred to 96-well microtiter plates containing $100 \mu \mathrm{l}$ RPMI $1640 /$ well. $50 \mu$ l of acidic poly aa at a final concentration of $1.4 \times 10^{-5} \mathrm{M}$ or RPMI 1640 alone were added followed within $5 \mathrm{~min}$ by $50 \mu \mathrm{l}$ of MBP at a final concentration of $1.4 \times 10^{-5} \mathrm{M}$ or controls (column buffer or RPMI 1640). MBP was not used at 5 $\times 10^{-6} \mathrm{M}$ as in K562 assays, because this concentration did not cause complete cessation of ciliary motion of tracheal ring epithelial cells 
over the assay period. An observer, unaware of the substance or combination being tested, examined the ring cultures at $24 \mathrm{~h}$ and $48 \mathrm{~h}$ using an inverted microscope. After final observations, specimens were fixed in $10 \%$ buffered formalin, embedded in paraffin, sectioned, and stained with hematoxylin and eosin.

\section{Criteria for damage to tracheal epithelium}

Our criteria for damage to the ciliated epithelium of the guinea pig tracheal rings were similar to those used previously (13). The term ciliostasis is used to describe the complete cessation of ciliary motion throughout the entire tracheal ring; "normal" tracheal rings had active, intact ciliary epithelium. At the time intervals when damage was assessed, ciliostasis was almost always accompanied by partial or complete exfoliation or "stripping" of the ciliated epithelial cells from the basement membrane of the mucosa with precipitates of MBP on or around the cells.

\section{Airway hyperresponsiveness and inhibition of hyperresponsiveness assays}

Animals. The animals used in this study were wild-caught adult male cynomolgus monkeys (Macaca fascicularis) weighing 3.5-7.5 kg. Each animal was housed individually in a specially designed open mesh cage and provided with food twice daily and water ad lib. Animals were fasted for $\sim 18 \mathrm{~h}$ before study.

Study protocol. Each animal was anesthetized with an intramuscular injection of ketamine $(4 \mathrm{mg} / \mathrm{kg})$ and xylazine $(1 \mathrm{mg} / \mathrm{kg})$, intubated with a cuffed endotracheal tube, and seated in an upright position in a specially designed support chair. Ketamine $(4 \mathrm{mg} / \mathrm{kg}$, i.m.) was used to supplement anesthesia when needed. Baseline respiratory system resistance (Rrs) was monitored for $15 \mathrm{~min}$ followed by methacholine doseresponse determinations $(15,16)$. Bronchoconstrictor response to inhaled methacholine was used to determine airway responsiveness. After completion of the methacholine dose-response, each animal received an aerosol treatment of either vehicle or polyglutamic acid ( $P$ $4886,61,200 \mathrm{~mol} \mathrm{wt}$; Sigma; this polyglutamic acid is a different lot and molecular weight from that of the P-4886 listed in Table I). Rrs was monitored for an additional $10 \mathrm{~min}$ after which each animal received an intratracheal injection of native MBP. Rrs was then monitored for 1 $h$ postinstillation after which the animals were allowed to recover from anesthesia. At $2 \mathrm{~h}$ postinstillation each animal was anesthetized (ketamine/xylazine), intubated, and methacholine dose-response determinations were performed. The study was designed so that the polyglutamic acid treatment experiments were bracketed by control (vehicle) treatment studies to ensure no change in MBP-induced effects over time.

Intratracheal MBP instillation. Native MBP was diluted in column buffer to a concentration of $200 \mu \mathrm{g} / \mathrm{ml}$ immediately before instillation. A total of $5 \mathrm{ml}$ (1 mg MBP) was slowly infused directly into the trachea through the endotracheal tube via a $20-\mathrm{cm}$ long piece of polyethylene 240 tubing attached to a 5-ml syringe. As control fluids, PBS and column buffer were tested and produced no effect on Rrs or methacholine sensitivity.

Rrs measurements. Respiratory system impedance was measured by discrete frequency sinusoidal forced oscillations superimposed on tidal breathing as previously described (15). The frequency range was computed to provide a single value representation of Rrs.

Aerosol delivery system. Aerosol inhalation treatments of vehicle or polyglutamic acid were administered by intermittent positive pressure breathing with a Bird Mark 7A respirator and micronebulizer (Mode 8158; Bird Products Corp., Palm Springs, CA). Each treatment consisted of 15 breaths per min (maximum inspiratory pressure $20 \mathrm{~cm}$ $\mathrm{H}_{2} \mathrm{O}$ ) for 6 min. Polyglutamic acid was dissolved in PBS at a concentration of $25 \mathrm{mg} / \mathrm{ml}$ just before use. Aerosol treatment delivered $\sim 1.5 \mathrm{mg}$ of polyglutamic acid to the lungs.

Methacholine dose-response determinations. Bronchial responsiveness was assessed by performing cumulative methacholine dose-response determinations as previously described (16). Briefly, after an initial aerosol challenge with vehicle (PBS), increasing concentrations of methacholine were administered until increases in Rrs of 100-200\% were obtained. Aerosol challenges were separated by $5-8 \mathrm{~min}$ or until Rrs returned to baseline values. Linear interpolation on a logarithmic scale was used to estimate the dose of methacholine at which a $100 \%$ increase in Rrs would have occurred (methacholine $\mathrm{PC}_{100}$ ).

Statistical analysis. Changes in Rrs and methacholine $\mathrm{PC}_{100}$ values were evaluated statistically with the use of Fischer's paired $t$ test. In each case, statistical significance was accepted when $P<0.05$.

\section{Inhibition of blood clotting}

To determine whether acidic poly aa inhibit blood clotting, stock concentrations of heparin, poly-L-glutamic acid $13,600 \mathrm{~mol}$ wt, poly-L-glutamic acid 77,800 mol wt, and/or MBP each at $1 \times 10^{-3} \mathrm{M}$ were added to $500 \mu \mathrm{l}$ whole blood from a normal human volunteer to final concentrations of $10^{-4} \mathrm{M}$ to $10^{-6} \mathrm{M}$ in $10 \times 75-\mathrm{mm}$ glass tubes and mixed. PBS and column buffer were used as controls. The tubes were checked at 1-min intervals and clotting time for each sample was recorded. After $30 \mathrm{~min}$, samples were checked every $30 \mathrm{~min}$ to a maximum of $15 \mathrm{~h}$.

\section{Results}

Inhibition of MBP toxicity to K562 cells by acidic poly aa. Various polyglutamic and polyaspartic aa were added to K562 cells followed by addition of MBP and cell viability was determined after $4 \mathrm{~h}$ (Table II). The poly aa ranged from 6,800 to 77,800 in mol wt (Table I) and were tested at concentrations from $1 \times 10^{-7} \mathrm{M}$ to $5 \times 10^{-6} \mathrm{M}$ for their ability to inhibit MBP toxicity at $5 \times 10^{-6} \mathrm{M}$. All of the acidic poly aa inhibited MBP toxicity at equimolar MBP concentrations (Table II). This inhibition was related to the acidic or anionic nature of the polymers because poly-L-asparagine at $5 \times 10^{-6} \mathrm{M}$ did not inhibit MBP toxicity. Also, some degree of polymerization was necessary for this effect because acidic amino acid monomers did not inhibit MBP toxicity. The acidic amino acids themselves were not toxic to the K562 cells at the concentrations tested.

As the concentration of the acidic poly aa was lowered below that of MBP, inhibition of MBP toxicity to the K562 cells was reduced (Table II). This reduction was not due to the type of acidic poly aa (glutamic versus aspartic or $D$ versus $L$ isomers) but was dependent upon the molecular weight and thus the degree of polymerization of the acidic aa. Because the degree of polymerization directly affects the total e of the acidic polymers, a comparison between the acidic poly aa concentrations necessary to balance the e of MBP at $5 \times 10^{-6} \mathrm{M}$ and the reduction in acidic poly aa inhibition of MBP toxicity to K562 cells was made (Tables I and II). At acidic polymer concentrations greater than or equal to those necessary to balance $e$ of MBP only poly-L-aspartic acid (11,500 mol wt) and poly-L-glutamic acid $(13,600 \mathrm{~mol} \mathrm{wt})$, both at $1 \times 10^{-6} \mathrm{M}$, were significantly different from controls; however, at acidic polymer concentrations less than those necessary to balance e of MBP, all polymers exhibited a drastic reduction in their ability to inhibit MBP toxicity. These data suggest that the e of an acidic poly aa is a critical determinant of its ability to inhibit MBP toxicity.

To better define the point at which acidic poly aa begin to effectively inhibit MBP toxicity, several of the acidic polymers were tested at $2.0,1.0$, and 0.5 times the concentration necessary to balance e of MBP at $5 \times 10^{-6} \mathrm{M}$ (Fig. 1). All four polymers tested inhibited MBP toxicity to K562 cells at a concentration of 2.0 times $e$ and were not significantly different from the medium control, although poly-DL-aspartic acid, the smallest polymer used in the study, was the least effective. At a 


\begin{tabular}{|c|c|c|c|c|c|c|c|}
\hline & \multirow[b]{3}{*}{ Mol wt } & \multicolumn{6}{|c|}{ Percent viability of K562 cells* } \\
\hline & & \multirow[b]{2}{*}{ Controls $^{\ddagger}$} & \multicolumn{5}{|c|}{ Native MPB $\left(5 \times 10^{-6} \mathrm{M}\right)$ added to aa concentration } \\
\hline & & & $1 \times 10^{-3} \mathrm{M}$ & $5 \times 10^{-6} \mathrm{M}$ & $1 \times 10^{-6} \mathrm{M}$ & $5 \times 10^{-7} \mathrm{M}$ & $1 \times 10^{-7} \mathrm{M}$ \\
\hline L-aspartic acid & 155 & $98.5 \pm 0.2$ & $3.4 \pm 4.7^{\S}$ & ND & ND & ND & ND \\
\hline L-glutamic acid & 169 & $98.8 \pm 0.2$ & $0.0 \pm 0.0^{5}$ & ND & ND & ND & ND \\
\hline Poly- $(\alpha, \beta)$-DL-aspartic acid & 6,800 & $97.0 \pm 2.7$ & ND & $\underline{90.1 \pm 6.7^{\prime \prime}}$ & $1.6 \pm 1.5$ & $6.0 \pm 3.9^{8}$ & $2.9 \pm 0.4^{\S}$ \\
\hline Poly (aspartic acid, glutamic acid) $1: 1$ & 9,000 & $98.4 \pm 1.4$ & ND & $\overline{95.5 \pm 0.9}$ & $22.0 \pm 19.7^{8}$ & $4.9 \pm 2.3^{8}$ & $0.0 \pm 0.0^{\S}$ \\
\hline Poly-L-aspartic acid & 11,500 & $98.0 \pm 2.4$ & ND & $\overline{87.5 \pm 7.9}$ & $\underline{84.3 \pm 1.6^{8}}$ & $22.7 \pm 7.2^{\S}$ & $2.7 \pm 3.8^{8}$ \\
\hline Poly-L-glutamic acid & 13,600 & $98.9 \pm 1.0$ & ND & $89.5 \pm 2.0$ & $\overline{72.9 \pm 0.7^{\S}}$ & $4.9 \pm 5.9^{\S}$ & $0.0 \pm 0.0^{\S}$ \\
\hline Poly-L-glutamic acid & 36,240 & $94.7 \pm 2.6$ & ND & $96.2 \pm 6.5$ & $\overline{96.9 \pm 1.8}$ & $\underline{67.5 \pm 20.4}$ & $1.3 \pm 1.8^{5}$ \\
\hline Poly-D-glutamic acid & 41,000 & $100.0 \pm 0.0$ & ND & $98.0 \pm 1.8$ & $99.0 \pm 1.7$ & $96.2 \pm 1.4$ & $11.2 \pm 0.9^{\S}$ \\
\hline Poly-L-aspartic acid & 42,500 & $97.4 \pm 2.3$ & ND & $100.0 \pm 0.0$ & $99.4 \pm 0.9$ & $\underline{100.0 \pm 0.0}$ & $8.7 \pm 1.4^{\S}$ \\
\hline Poly-D-glutamic acid & 66,000 & $93.4 \pm 6.1$ & ND & $96.4 \pm 1.7$ & $97.3 \pm 1.0$ & $9 \underline{90.6 \pm 3.4}$ & $0.0 \pm 0.0^{\S}$ \\
\hline Poly-L-glutamic acid & 77,800 & $95.1 \pm 3.8$ & ND & $93.9 \pm 8.0$ & $95.5 \pm 6.4$ & $\overline{95.4 \pm 3.2}$ & $2.6 \pm 3.5^{8}$ \\
\hline Poly-L-asparagine & 10,400 & $85.3 \pm 5.1$ & ND & $5.9 \pm 9.5^{\S}$ & $1.2 \pm 2.1^{5}$ & $0.0 \pm 0.0^{\S}$ & $0.0 \pm 0.0^{\S}$ \\
\hline Native MBP & 13,801 & $1.0 \pm 3.2$ & & & & & \\
\hline Column buffer & & $96.7 \pm 1.6$ & & & & & \\
\hline Medium & & $96.5 \pm 3.7$ & & & & & \\
\hline
\end{tabular}

* Values are mean percent viability \pm 1 SD of one to three 4-h experiments, each consisting of duplicate wells. ${ }^{\ddagger}$ Controls consist of aa, acidic poly aa, poly-L-asparagine, native MBP, column buffer, or medium tested alone. All controls at $5 \times 10^{-6} \mathrm{M}$ except L-aspartic and L-glutamic acid monomers which were tested at $1 \times 10^{-3} \mathrm{M} .{ }^{8} P<0.001$ for values tested against appropriate nontoxic aa control using Student's $t$ test; MBP added within $5 \mathrm{~min}$ after addition of acidic aa. "Values underlined are from acidic poly aa test concentrations closest to but not less than concentration for balanced charge (Table I) for a particular acidic poly aa.

concentration of 1.0 times e the polyaspartic acids were significantly different from the controls while the polyglutamic acids were not; mean percent viability was lowest for poly-DL-aspartic acid (23.9 \pm 4.2$)$ and highest for poly-L-glutamic acid 77,800 mol wt (89.1 \pm 2.3$)$. All of the polymers were significantly different from the medium control at a concentration of 0.5 times $\mathrm{e}$ and afforded little protection against MBP. Thus, acidic poly aa begin to effectively protect K 562 cells against MBP toxicity at concentrations between 1.0 and 2.0 times e.

In the first set of experiments, the acidic poly aa were added to the K562 cells before MBP. The ability of the acidic poly aa

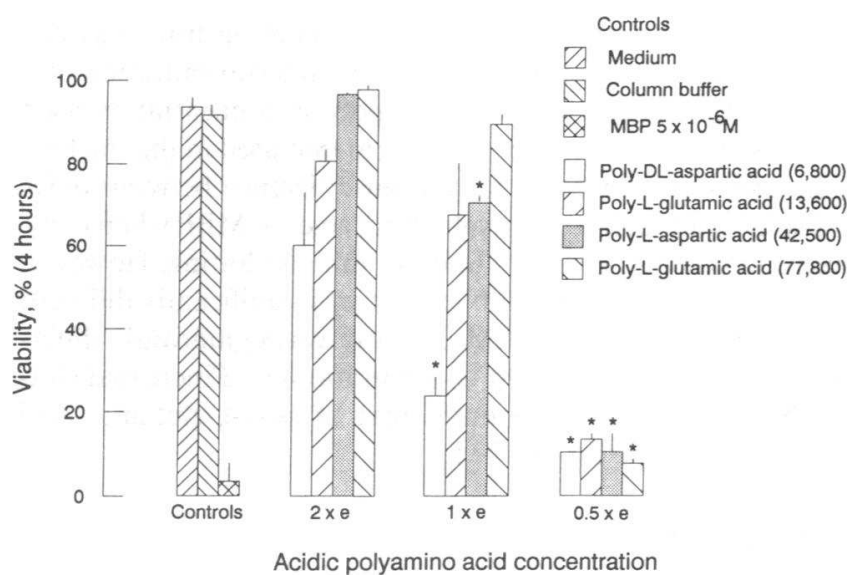

Figure 1. Acidic poly aa inhibition of MBP toxicity to K562 cells at 2, 1 , and 0.5 times acidic poly aa concentration necessary to balance e of MBP at $5.0 \times 10^{-6} \mathrm{M}$. Values are mean percent $\pm 1 \mathrm{SD}$ of one experiment consisting of duplicate wells. ${ }^{*} P<0.01$ for values tested against appropriate controls using Student's $t$ test. to inhibit MBP toxicity after MBP had already been added to the cells was tested and the data are shown in Fig. 2. Acidic poly aa concentrations which were effective in inhibiting MBP toxicity previously were not as effective in inhibiting MBP toxicity when MBP was added to the cells before the aa polymers. There is some protection when the acidic poly aa are added within 15 min after MBP addition. However, little protection is seen when the polymers are added one or more hours after MBP addition because 90\% of cells are killed within $30 \mathrm{~min}$ of incubation with MBP. Only poly-L-glutamic acid $(77,800$ mol wt) at equimolar MBP concentration of $5 \times 10^{-6} \mathrm{M}$ added

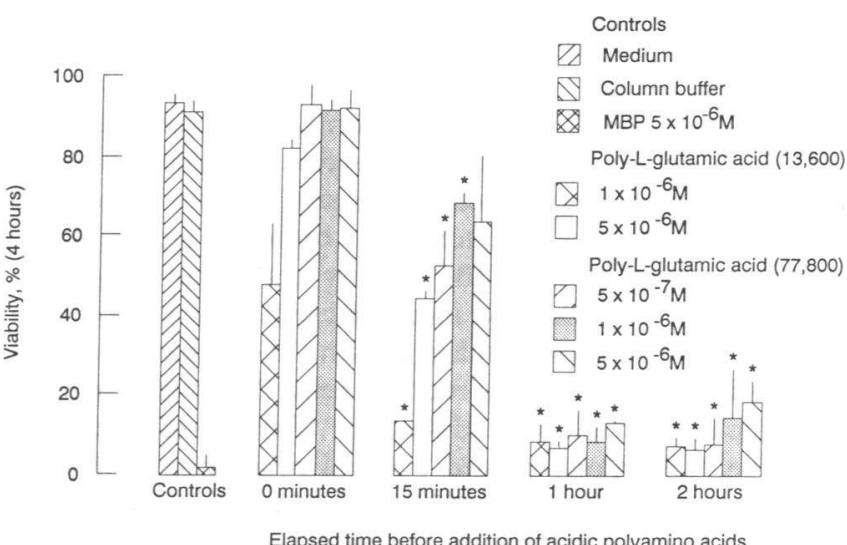

Figure 2. Acidic poly aa inhibition of MBP toxicity to K562 cells over time. Acidic polymers were added before MBP $(0 \mathrm{~min})$ or $15 \mathrm{~min}$, $1 \mathrm{~h}$, or $2 \mathrm{~h}$ after addition of MBP to cell cultures. Values are mean percent viability \pm 1 SD of one to two experiments consisting of duplicate wells. ${ }^{*} P<0.05$ values tested against acidic poly aa added at 0 min using Student's $t$ test. 


\begin{tabular}{|c|c|c|c|c|c|c|}
\hline & \multirow[b]{3}{*}{ Mol wt } & \multicolumn{5}{|c|}{ Percent viability of $\mathrm{K} 562$ cells* } \\
\hline & & \multirow{2}{*}{$\frac{\text { Controls }^{\ddagger}}{5 \times 10^{-6} \mathrm{M}}$} & \multicolumn{4}{|c|}{ Poly-L-arginine or ECP (both at $5 \times 10^{-6} \mathrm{M}$ ) added to acidic poly aa concentrations of } \\
\hline & & & $5 \times 10^{-6} \mathrm{M}$ & $1 \times 10^{-6} \mathrm{M}$ & $5 \times 10^{-7} \mathrm{M}$ & $1 \times 10^{-7} \mathrm{M}$ \\
\hline Poly-L-glutamic acid & 13,600 & $96.9 \pm 4.5$ & $99.1 \pm 1.3^{8}$ & $4.9 \times 0.3^{\prime \prime}$ & $0.0 \pm 0.0^{11}$ & $0.0 \pm 0.0^{\prime \prime}$ \\
\hline Poly-L-glutamic acid & 77,800 & $97.2 \pm 2.5$ & $97.9 \pm 3.0$ & $\underline{97.2 \times 4.0}$ & $22.9 \pm 3.0^{\prime \prime}$ & $2.0 \pm 2.9^{\prime \prime}$ \\
\hline Poly-L-arginine hydrochloride & 12,000 & $0.0 \pm 0.0$ & & & & \\
\hline Medium & & $97.8 \pm 1.0$ & & & & \\
\hline Poly-L-glutamic acid & 13,600 & $100.0 \pm 0.0$ & $77.2 \pm 4.9^{11}$ & $\underline{73.7 \times 5.8}$ & $50.2 \pm 1.3^{\prime \prime}$ & $59.8 \pm 20.6$ \\
\hline Poly-L-glutamic acid & 77,800 & $100.0 \pm 0.0$ & $98.3 \pm 0.5$ & $96.4 \times 5.2$ & $\underline{91.2 \pm 3.3}$ & $58.4 \pm 11.8^{\| \prime}$ \\
\hline ECP & 16,000 & $35.7 \pm 6.2$ & & & & \\
\hline Medium & & $86.8 \pm 2.1$ & & & & \\
\hline
\end{tabular}

* Values are mean percent viability \pm 1 SD of one 4-h experiment consisting of duplicate wells for poly-L-arginine hydrochloride and a second experiment consisting of duplicate wells for ECP. ${ }^{\ddagger}$ Controls consist of acidic poly aa, poly-L-arginine, ECP, or medium tested alone. ${ }^{8}$ Values underlined are from acidic poly aa test concentrations closest to but not less than concentration for balanced charge (Table I) for a particular acidic poly aa. " $P<0.05$ for values tested against appropriate acidic poly aa controls using Student's $t$ test; poly-L-arginine hydrochloride or ECP added within 5 min after addition of acidic poly aa.

15 min after MBP was not significantly different from its control. Increasing acidic poly aa concentrations 10 -fold to 5 $\times 10^{-5} \mathrm{M}$ (data not shown) at all addition times had no significant effect. Apparently, the acidic poly aa have little effect on K562 viability unless they are added before or immediately after addition of $5 \times 10^{-6} \mathrm{M}$ MBP.

Inhibition of poly-L-arginine and ECP toxicity to $\mathrm{K} 562$ cells by acidic poly $a a$. To determine if this toxic inhibition by acidic poly aa is limited only to MBP or may apply to other cationic toxins, acidic poly aa were tested for their ability to inhibit poly-L-arginine and ECP toxicity to K562 cells. Both poly-L-arginine and ECP toxicity were inhibited by acidic poly aa (Table III). Generally, there were no significant differences between test concentrations and controls when acidic poly aa test concentrations were at least equal to the concentration needed for a balanced e for poly-L-arginine or ECP except for poly-L-glutamic acid 13,600 mol wt tested against ECP. Below acidic poly aa concentrations necessary for a balanced $e$, inhibition of toxicity was greatly reduced for all acidic poly aa tested. Because these acidic poly aa inhibit poly-L-arginine and ECP toxicity, this mechanism is not specific to MBP and may apply to numerous cationic toxins.

Inhibition of MBP toxicity to tracheal ring epithelium by acidic poly aa. Acidic poly aa were used as antagonists of MBP toxicity to guinea pig tracheal epithelium as a model for their role as an inhibitor of MBP toxicity in bronchial asthma. Polyglutamic acids inhibited MBP toxicity to the tracheal epithelium (Fig. 3). Tracheal rings incubated with MBP alone exhibited gross morphologic damage at $24 \mathrm{~h}$. The damage included ciliostasis and exfoliation of mucosal cells. Precipitates of MBP were apparent on and around the cells. After $48 \mathrm{~h}$ of incubation only the MBP alone-treated tracheal rings showed damage (Fig. 3, $A$ and $B$ ). The tracheal rings treated with acidic polyglutamic acids or a combination of polyglutamic acids and MBP appeared normal with active cilia (Fig. 3, $C$ and $D$ ). Some of the cilia of the rings treated with a combination of polyglutamic acids and MBP appeared to have a small amount of precipitate on them which may be polyglutamic acid and/or MBP (Fig. 3 $D$ ). There was no detectable damage to the cilia associated with this precipitate.
Inhibition of MBP-induced effects on the airway by polyglutamic acid. MBP instillation resulted in an immediate increase in Rrs that peaked between 5 and 10 min postinstillation and resolved by $1 \mathrm{~h}$ (Fig. 4). Pretreatment with polyglutamic acid significantly inhibited the MBP-induced increase in Rrs. Polyglutamic acid treatment by itself did not alter Rrs (data not shown).

The effects of MBP instillation on airway responsiveness to inhaled methacholine during control and polyglutamic acid treatment studies are shown in Fig. 5. MBP administration alone resulted in a dramatic increase in airway responsiveness as indicated by a decrease in calculated methacholine $\mathbf{P C}_{100}$ values. Polyglutamic acid pretreatment significantly inhibited the MBP-induced increase in airway responsiveness in each animal studied.

Effect of acidic poly a on blood clotting. Although heparin is an effective antagonist of MBP toxicity $(1,17)$, its possible clinical use in bronchial asthma could be limited by its ability to inhibit blood clotting. To determine if acidic poly aa inhibit blood clotting, whole blood was incubated with heparin, polyglutamic acids, and/or MBP, and the clotting time was observed. These data are shown in Fig. 6. At a concentration of 1 $\times 10^{-5} \mathrm{M}$, twice the highest acidic poly aa concentration used in the K562 assays and comparable to that used in the tracheal ring assays, there was no significant difference between polyglutamic acids alone or polyglutamic acids + MBP when compared to controls in their ability to inhibit clotting. However, poly-L-glutamic acid $77,800 \mathrm{~mol}$ wt was significantly different from controls at $1 \times 10^{-4} \mathrm{M}$. Heparin was significantly different from controls at all test concentrations and increased clotting time at least to 45 times that of poly-L-glutamic acid $77,800 \mathrm{~mol}$ wt at $1 \times 10^{-4} \mathrm{M}$.

\section{Discussion}

The mechanism by which MBP kills cells is unknown. It has been suggested that the cationic charge of MBP may attract it to the anionic charged cell surface of targets, whereupon the apolar residues insert into and perturb the lipid milieu (2). MBP likely damages cell membranes $(3,4)$ which increases 

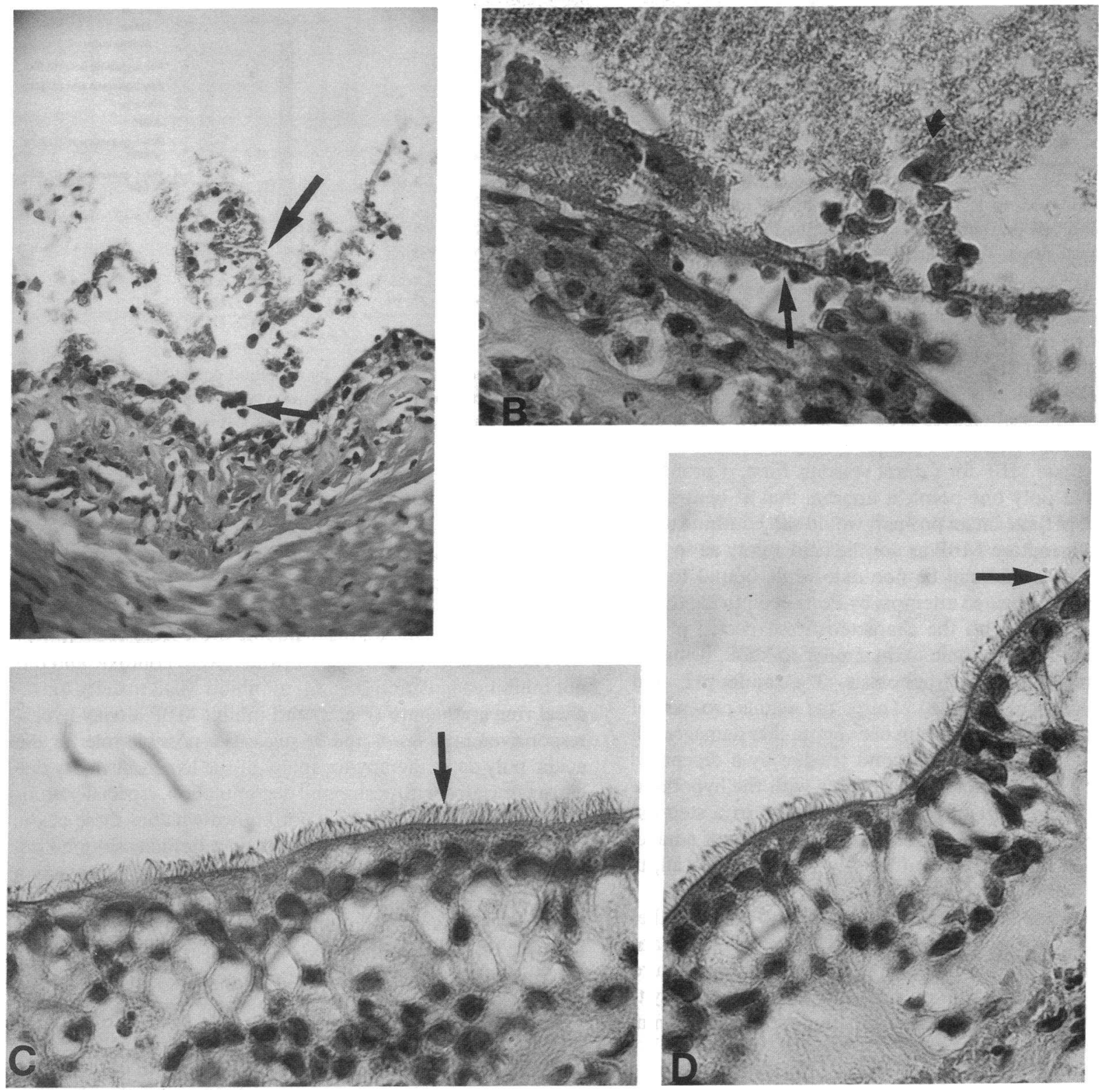

Figure 3. Effect of acidic poly aa on MBP toxicity to guinea pig tracheal rings by histologic examination. Photomicrographs of fixed tissue, stained with hematoxylin-eosin, and examined by light microscopy. $(A)$ Treatment with MBP alone. Arrows indicate exfoliation and destruction of ciliated epithelial cells. $(B)$ Treatment with MBP alone. Straight arrow indicates exfoliated cells. Note MBP precipitate on and around exfoliated cells (curved arrow). (C) Treatment with poly-L-glutamic acid mol wt 77,800 alone. Ciliated epithelium (arrow) appears intact. (D) Treatment with poly-L-glutamic acid mol wt 77,800 and MBP. Ciliated epithelium appears intact. Note particulate matter (arrow) on some cilia which may be precipitates of polyglutamic acid and/or MBP. $(A) \times 225 ;(B-D) \times 560$.

their permeability without concurrent formation of transmembrane channels (3).

In this study MBP toxicity to K562 cells and guinea pig tracheal epithelium was inhibited by acidic poly aa. This inhibition was directly related to the ability of negatively charged acidic poly aa to balance and thus neutralize the positively charged MBP. The ratio of the absolute values of the predicted e for the acidic pro-part of proMBP to the predicted e of cationic MBP is 1.2. Inhibition of MBP toxicity by acidic poly aa at concentrations above that necessary to balance charge (ratio of 1.0) but not significantly at concentrations below that necessary to balance charge (Table II; Fig. 1) suggests that the acidic pro-portion of proMBP functions to mask MBP toxicity. Thus, it is likely that proMBP is a nontoxic molecule that protects the eosinophil from damage while the protein is processed through the endoplasmic reticulum to its sequestered site in the eosinophil granule as toxic MBP.

These data also show that the acidic poly aa must be added before or immediately after addition of $5 \times 10^{-6} \mathrm{M}$ MBP to inhibit its toxicity (Fig. 2). Presumably, when MBP is added before the acidic polymers and allowed to incubate with the target for a short period of time, MBP will bind to the target membranes and will not be effectively inhibited by the acidic polymers after that time; addition of acidic poly aa before MBP 


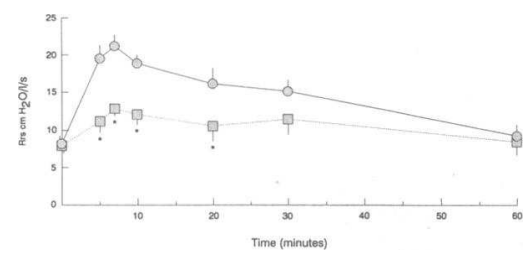

MBP-induced increases in Rrs. Circles represent MBP alone instillation. Squares indicate polyglutamic acid inhalation followed in 10 min by MBP instillation. Values are mean \pm SEM, $n=5 .{ }^{*} P<0.05$.

may allow them to bind to MBP preventing it from binding to the target membranes.

This has implications in the processing of proMBP. The presumed enzyme that cleaves proMBP is unknown. It is unlikely that this enzyme cleaves proMBP only at one point into pro-part and toxic MBP for several reasons. First, if proMBP were cleaved at only one point, it appears that at neutral or nearly neutral $\mathrm{pH}$, the intact pro-part would still continue to be associated with mature MBP as are the acidic poly aa in this study and MBP would still be nontoxic while bound to the pro-part. Second, repeated attempts by Ponz et al. to identify a small acidic protein with the characteristics expected of the pro-part of BTAH, a cationic toxin similar to MBP, using gel filtration, preparative electrophoresis at alkaline $\mathrm{pH}$, and HPLC were unsuccessful (18). Third, the acidic pro-part of promellitin, a cationic toxin from bee venom that damages cell membranes, is cleaved every second residue by a dipeptidyl peptidase (19). These data are in keeping with the hypothesis that the acidic pro-part of proMBP is cleaved in a stepwise fashion that results in toxic MBP and a number of smaller peptides which do not bind sufficiently to MBP, if at all, to inhibit its toxicity.

Polyarginine and ECP are potent toxins and although they may kill targets differently from $\operatorname{MBP}(3,20)$, polyarginine and ECP share with MBP the property of a highly cationic nature. Acidic poly aa inhibition of polyarginine and ECP toxicity to K562 cells (Table III) may reflect the binding of polyarginine

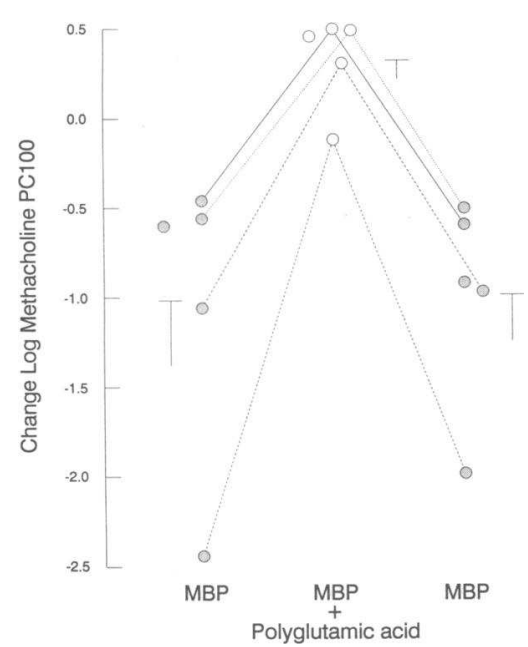

Figure 5. Changes in methacholine $\mathrm{PC}_{100}$ values $2 \mathrm{~h}$ post-MBP instillation during control studies (MBP alone; shaded circles) and polyglutamic acid pretreatment studies (empty circles) for each of five animals. Bars represent mean \pm SEM, $n=5$, for each treatment group. Polyglutamic acid treatment experiments were bracketed by control (MBP alone) treatment studies to ensure no change in MBP-induced effects

over time. Animals were rested for a minimum of 3 wk between treatments. MBP-induced increases in airway responsiveness were significantly inhibited in each animal.

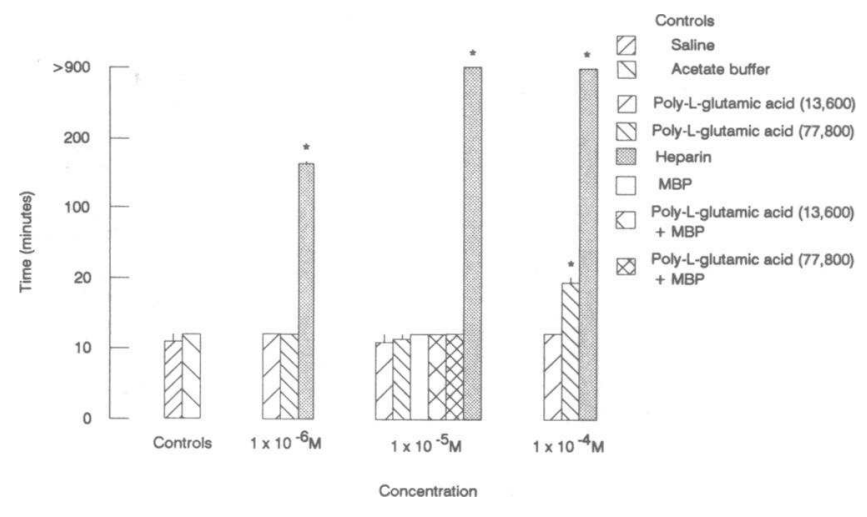

Figure 6. Effects of acidic poly aa on blood coagulation. Values are mean coagulation time \pm 1 SD of one to two experiments in triplicate. ${ }^{*} P<0.001$ for values tested against saline control using Student's $t$ test.

and ECP to the acidic poly aa, precluding the binding of these toxins to target cells. Because this inhibition is not restricted to MBP but also applies to ECP and polyarginine, treatment of target cells with acidic poly aa may perturb the toxic activities of numerous cationic toxins including those from eosinophils.

The ability of acidic poly aa (which were nontoxic and did not inhibit coagulation [Fig. 6]) to inhibit MBP toxicity to tracheal ring epithelium (Fig. 3) and inhibit MBP airway hyperresponsiveness (Figs. 4 and 5) suggests a possible role for the acidic poly aa as therapy for many of the hypersensitivity diseases associated with eosinophil degranulation, especially bronchial asthma. Presently, it is not known whether these acidic poly aa would inhibit MBP and other cationic eosinophil degranulation products in vivo. Heparin, which has been shown to inhibit MBP toxicity $(1,17)$, does inhibit whole eosinophil toxicity to Trypanosoma cruzi trypomastigotes (21). Studies are underway to test the effectiveness of acidic poly aa to inhibit airway hyperresponsiveness associated with antigen induced asthma in the primate model.

\section{Acknowledgments}

We thank Linda $\mathrm{H}$. Arneson for preparation of the manuscript.

This work was supported in part by grants from the National Institutes of Health, AI-07047, AI-15231 and AI-09728, and by the Mayo Foundation.

\section{References}

1. Gleich, G. J., and C. R. Adolphson. 1986. The eosinophilic leukocyte: structure and function. Adv. Immunol. 39:177-253.

2. Wasmoen, T. L., M. P. Bell, D. A. Loegering, G. J. Gleich, F. G. Prendergast, and D. J. McKean. 1988. Biochemical and amino acid sequence analysis of human eosinophil granule major basic protein. J. Biol. Chem. 263:12559-12563.

3. Young, J. D.-E., C. G. B. Peterson, P. Venge, and Z. A. Cohn. 1986. Mechanism of membrane damage mediated by human eosinophil cationic protein. Nature (Lond.). 321:613-616.

4. Kroegel, C., U. Costabel, and H. Matthys. 1987. Mechanism of membrane damage mediated by eosinophil major basic protein. Lancet. i:1380-1381.

5. Gundel, R. H., L. G. Letts, and G. J. Gleich. 1991. Human eosinophil major basic protein induces airway constriction and airway hyperresponsiveness in primates. J. Clin. Invest. 87:1470-1473.

6. Barker, R. L., G. J. Gleich, and L. R. Pease. 1988. Acidic precursor revealed in human eosinophil granule major basic protein cDNA. J. Exp. Med. 168:14931498. 
7. Barker, R. L., D. A. Loegering, R. M. Ten, K. J. Hamann, L. R. Pease, and G. J. Gleich. 1989. Eosinophil cationic protein cDNA: comparison with other toxic cationic proteins and ribonucleases. J. Immunol. 143:952-955.

8. Pineda, A. A., S. M. Brzica, Jr., and H. F. Taswell. 1977. Continuous- and semicontinuous flow blood centrifugation systems: therapeutic applications, with plasma-, platelet-, lympha-, and eosinoapheresis. Transfusion (Phila.). 17:407416.

9. Gleich, G. J., A. A. Pineda, G. O. Solley, and H. F. Taswell. 1981. Cytapheresis for eosinophilia. In Proceedings of the Workshop on Therapeutic Plasmapheresis and Cytapheresis. G. J. Nemo and H. Taswell, editors. U. S. Department of Health and Human Services, NIH Publication No. 82-1665. 73-79.

10. Gleich, G. J., D. A. Loegering, K. G. Mann, and J. E. Maldonado. 1976. Comparative properties of the Charcot-Leyden crystal protein and the major basic protein from human eosinophils. J. Clin. Invest. 57:633-640.

11. Ackerman, S. J., D. A. Loegering, P. Venge, I. Olsson, J. B. Harley, A. S. Fauci, and G. J. Gleich. 1983. Distinctive cationic proteins of the human eosinophil granule: major basic protein, eosinophil cationic protein, and eosinophil-derived neurotoxin. J. Immunol. 131:2977-2982.

12. Nakajima, H., D. A. Loegering, and G. J. Gleich. 1988. Cytotoxicity of eosinophil granule proteins for tumor cells. FASEB (Fed. Am. Soc. Exp. Biol.). J. 2:A811. (Abstr.)

13. Motojima, S., E. Frigas, D. A. Loegering, and G. J. Gleich. 1989. Toxicity of eosinophil cationic proteins for guinea pig tracheal epithelium in vitro. $\mathbf{A m}$. Rev. Respir. Dis. 139:801-805.
14. Lane, B. P., S. L. Miller, and E. J. Drummond. 1976. Use of tracheal organ cultures in toxicity testing. Environ. Health Perspect. 16:89-98.

15. Wegner, C. D., A. C. Jackson, J. D. Berry, and J. R. Gillespie. 1984. Dynamic respiratory mechanics in monkeys measured by forced oscillations. Respir. Physiol. 55:47-61.

16. Gundel, R. H., M. E. Gerritsen, G. J. Gleich, and C. D. Wegner. 1990. Repeated antigen inhalation results in a prolonged airway eosinophilia and airway hyperresponsiveness in primates. J. Appl. Physiol. 68:779-786.

17. Butterworth, A. E., D. L. Wassom, G. J. Gleich, D. A. Loegering, and J. R. David. 1979. Damage to schistosomula of Schistosoma mansoni induced directly by eosinophil major basic protein. J. Immunol. 122:221-229.

18. Ponz, F., J. Paz-Ares, C. Hernandez-Lucas, F. Garcia-Olmedo, and P. Carbonero. 1986. Cloning and nucleotide sequence of a cDNA encoding the precursor of the barley toxin $\alpha$-hordothionin. Eur. J. Biochem. 156:131-135.

19. Kreil, G., L. Haiml, and G. Suchanek. 1980. Stepwise cleavage of the pro part of promelittin by dipeptidylpeptidase IV. Eur. J. Biochem. 111:49-58.

20. Kierszenbaum, F., S. J. Ackerman, and G. J. Gleich. 1981. Destruction of bloodstream forms of Trypanosoma cruzi by eosinophil granule major basic protein. Am. J. Trop. Med. Hyg. 30:775-779.

21. Kierszenbaum, F., S. J. Ackerman, and G. J. Gleich. 1982. Inhibition of antibody-dependent eosinophil-mediated cytotoxicity by heparin. J. Immunol. 128:515-517. 